\title{
DESENVOLVIMENTO NEUROPSICOMOTOR E
} AFETIVO-SOCIAL DE BEBÊS COM FISSURA

\section{LABIOPALATINA RELACIONADO AOS ESTADOS DE HUMOR MATERNO.}

Mayara dos Santos Baldin ${ }^{1}$

Mestranda em ciências pelo HRAC/USP E-mail:

mayarabaldin@gmail.com

\section{Ana Luiza Martins}

Apolônio ${ }^{2}$

Mestranda em ciências pela FOB/USP. E-mail:

analuiza.apolonio@gmail.com

\section{Anaí Ramos Vieira ${ }^{3}$}

Residente em síndromes e anomalias craniofaciais pelo HRAC/USP E-mail:

anai.vieira@hotmail.com

Cibele Nunes Moretti ${ }^{4}$

Especialista em psicologia pelo HRAC/USP E-mail:

cib_psico@yahoo.com.br

Maria de Lourdes Erigi Tabaquim $^{5}$

Professora Livre-docente do Departamento de

Fonoaudiologia da FOB-USP e da Pós-graduação em Ciências da Reabilitação do HRAC-USP. E-mail: malu.tabaquim@usp.br

\author{
NEUROPSICOMOTOR AND SOCIAL AFFECTIVE \\ DEVELOPMENT OF BABIES WITH CLEFT LIP/PALATE \\ RELATED TO MATERNAL MOOD.
}

\section{RESUMO}

O diagnóstico de fissuras labiopalatais geralmente desencadeia dificuldades no estabelecimento do vínculo mãe-bebê, importante fator para o desenvolvimento infantil pleno. Assim, buscou-se analisar a interferência da depressão e ansiedade maternas no desenvolvimento psicomotor e emocional-afetivo de bebês com fissura labiopalatina. Participaram 40 díades mãe-bebê. Para avaliação da depressão utilizou-se o Inventário de Depressão de Beck (BDI) e para ansiedade, o Inventário de Ansiedade de Beck (BAI). Na avaliação do desenvolvimento neuropsicomotor (DNPM) dos bebês utilizou-se o Protocolo de Avaliação do Desenvolvimento Global de Crianças no Primeiro ano de Vida e para a retração, a Escala de Avaliação da Reação de Retração Prolongada da Criança Pequena (BADS). Os resultados evidenciaram valores mínimos de depressão (72,5\%) e ansiedade (60\%). O DNPM apresentou-se dentro da normalidade, $84,7 \%$ (DP: 4,39 ) dos marcos do desenvolvimento infantil. A BADS indicou que $60 \%$ das crianças apresentaram boa qualidade afetivo-emocional, $27,5 \%$ apresentaram comportamento de risco e $12,5 \%$ déficit intenso. Não houve correlação entre nível de depressão materna e o nível de desenvolvimento global do bebê. Houve correlação positiva entre ansiedade materna e a quantidade de gestos de autoestimulação realizada pelo bebê $(r=0,40)$. Conclui-se que 0 desenvolvimento afetivo-emocional de crianças com fissura labiopalatina não foi afetado pelos níveis de depressão materna, mas o aumento na frequência de gestos de autoestimulação está relacionado a altos níveis de ansiedade materna. O DNPM não foi afetado pelo estado de saúde mental materna, indicando que estar em um ambiente de cuidado institucional pode atuar como um fator de proteção para o desenvolvimento infantil.

PALAVRAS-CHAVE: Fissura labial. Fissura palatina. Desenvolvimento infantil. Saúde materno-infantil. 


\section{ABSTRACT}

When diagnosed by the family, cleft lip/palate trigger reactions such as that may hinder the establishment of the mother-baby bond, which is important for emotional and cognitive child development.Thus, it sought to analyze the interference of maternal depression and anxiety in the psychomotor and emotional-affective development of infants with cleft lip and palate. 40 motherinfant dyads were evaluated. The Beck Depression Inventory and the Beck Anxiety Inventory were used for assessment of maternal depression and axiety. Neuropsychomotor and affective-emotional development were assessed by the Global Child Development Assessment Protocol and by the Baby Alarm Distress Scale (BADS). Minimum values of depression $(72.5 \%)$ and anxiety $(60 \%)$ were prevalent. Neuropsychomotor development was normal, with $84.7 \%$ (SD: 4.39) of the children developmental milestones by age reached by the participants. BADS indicated that $60 \%$ of the children presented good affective-emotional quality, $27.5 \%$ had a risk behavior and $12.5 \%$ presented an intense deficit. There was no correlation between maternal depression levels and the overall developmental level of the infant. But there was a positive correlation between maternal anxiety leval and self stimulation gestures made by the baby $(r=0,40)$. This study concluded that children with cleft lip and palate affective-emotional development were not affected by maternal depression levels, but the increase in the frequency of self-stimulation gestures are related to high levels of maternal anxiety. Neuropsychomotor development was not affected by maternal mental health status, indicating that being in a institutional care setting can act as a protective factor for children development.

KEYWORDS: Cleft lip. Cleft palate. Child development. Maternal and child health.

\section{INTRODUÇÃO}

As fissuras labiopalatinas são malformações que ocorrem quando há a fusão incompleta dos processos faciais no final do período embrionário e início do período fetal (SILVA FILHO et al., 1998). É a anomalia orofacial mais frequente, tendo incidência sujeita a alta variação étnica e geográfica, sendo aceita a taxa de 1:650 nascidos vivos no Brasil (FREITAS et al., 2012). Aproximadamente $70 \%$ dos casos surgem de maneira isolada, sendo chamadas de fissuras labiopalatinas não-sindrômicas (FLPNS), e os outros 30\% estão associados a outras malformações, como parte de um quadro sindrômico (FREITAS et al., 2012). A FLPNS pode acometer diferentes estruturas faciais e sua classificação se dá a partir da localização anatômica, podendo ser pré-forame (apenas lábio), transforame (lábio e palato) e pós-forame (apenas palato) (TRINDADE; SILVA FILHO, 1998; GARIB et al., 2011).

A etiologia das FLPNS é multifatorial, sendo influenciada tanto por fatores genéticos, quanto ambientais. Os fatores de risco ambientais envolvem aspectos nutricionais, o uso de medicamentos anticonvulsivantes, fumo passivo e ativo, hipertermia, estresse, obesidade materna, exposição à radiação e à infecções durante a gravidez (COUTINHO, 2009). Estudos em gêmeos mostram até $50 \%$ de concordância na ocorrência da fissura em homozigóticos e $8 \%$ em dizigóticos, indicando que, ainda que haja um fator ambiental, o componente genético das fissuras é bastante influente (GROSEN et al., 2011). Além disso, o 
risco de recorrência familial em famílias com pessoas afetadas é muito alto quando comparado ao da população sem parentes afetados, variando entre $4,1 \%$ a $0,3 \%$, contra a incidência de $0,1 \%$ na população geral (GROSEN et al., 2011).

O desenvolvimento infantil é um processo multidimensional e integral, que se inicia com a concepção e que engloba o crescimento físico, a maturação neurológica, o desenvolvimento comportamental, sensorial, cognitivo e de linguagem, assim como as relações socioafetivas (ORGANIZAÇÃO PANAMERICANA DA SAÚDE, 2005). Além disso, é resultado da interação da maturação do sistema nervoso central (SNC) e de fatores biológicos, relacionais, afetivos, simbólicos, contextuais e ambientais.

Os primeiros anos de vida são considerados críticos para o desenvolvimento das habilidades motoras, cognitivas e sensoriais, já que é neste período que ocorre a maturação do sistema nervoso central, na qual o cérebro se desenvolve mais rapidamente (PANCERI; PEREIRA; VALENTINI, 2017). Essa é considerada a fase ótima da plasticidade neural na qual abrem-se oportunidades para o desenvolvimento de funções importantes para uma boa saúde no futuro (UNICEF, 2015). Apesar das individualidades do desenvolvimento que cada criança expressa no modo como age, reage e interage com objetos, pessoas, situações e ambientes, existem marcos do desenvolvimento nos primeiros 03 anos de vida que indicam um desenvolvimento saudável. A aquisição desses marcos depende do funcionamento orgânico adequado e da quantidade e qualidade dos estímulos ambientais que a criança recebe (MATTA, 2001).

No período sensório-motor a criança tem pouco controle sobre o seu corpo e seus movimentos. Durante os dois primeiros meses de vida começa a sustentar o pescoço e, aos quatro meses, com ajuda já consegue sentar. O rastejar e o engatinhar aparecem como as primeiras tentativas de movimentação intencional, por volta dos quatro/seis meses; aos sete, espera-se que a criança consiga sentar-se sem qualquer auxílio; aos oito/nove, ficar em pé com apoio; e aos onze meses, andar com ajuda. A função de andar é adquirida gradativamente quanto ao seu controle e harmonia para o a conquista da habilidade autônoma. Ao aprender a andar, a criança liberta as mãos para outras descobertas e aprendizagens, condição importante para a exploração e desenvolvimento de competências cognitivas (MATTA, 2001; RIBEIRO; PEROSA; PADOVANI, 2014)

As transformações no primeiro ano de vida ocorrem nas diferentes áreas do desenvolvimento concomitantemente, porém, obedecendo diferentes ritmos e aquisições em cada domínio. A linguagem e a fala são manifestadas por comportamentos verbais, como o balbucio e choro, e os não verbais, como 0 olhar, expressões faciais, toque, entre outros, até surgirem as primeiras palavras. Esta crescente capacidade linguística traz inúmeras implicações ao nível da comunicação com o meio (PAPALIA; OLDS; FELDMAN, 2001).

Segundo Brazelton (2006), uma criança entre as seis e as oito semanas utiliza o sorriso como um meio para captar a atenção dos seus pais (linguagem nãoverbal). Para além do sorriso, o choro é uma das principais formas da criança se comunicar (MATTA, 2001). A capacidade de expressar sentimento, como ser carinhosa, de depositar maior confiança nas figuras de referência e de estabelecer intimidade, aumenta entre os quatro e seis meses (BRAZELTON, 2006). Com um ano de idade a criança ainda se mostra muito dependente emocionalmente da mãe, contudo, ao longo da primeira infância, já começa a se diferenciar e expressar vontade própria, o que a permite vivenciar diferentes experiências e enriquecer o seu repertório emocional. O processo de 
desenvolvimento toma em si as singularidades humanas, as especificidades hereditárias do indivíduo e aquelas que são resultantes da sua experiência de interação com a realidade social e física. O processo de desenvolvimento da criança é um processo pessoal, único, situado num contexto histórico e cultural que, também, o influencia. A criança desenvolve-se em diferentes ambientes, mais ou menos familiares, que lhe oferecem as suas primeiras experiências de vida (PAPALIA; OLDS; FELDMAN, 2001).

Entende-se, portanto, que dos 0 aos 3 anos o desenvolvimento do indivíduo está mais vulnerável a transformações de acordo com os fatores negativos ou positivos aos quais é exposto, assim fatores endógenos e exógenos que perturbem o desenvolvimento podem provocar, com maior ou menor intensidade, transtornos nesse processo (MINISTÉRIO DA SAÚDE, 2016). Portanto, tão importante quanto conhecer o desenvolvimento típico, se faz o conhecimento dos fatores interferentes nesse desenvolvimento. Dentre os fatores de risco para transtornos no desenvolvimento infantil estão: prematuridade, asfixia perinatal, hemorragia periventricular, displasia broncopulmonar, distúrbios bioquímicos do sangue (hipoglicemia, policitemia e hiperbilerrubinemia), malformações, infecções congênitas ou perinatais (toxoplasmose, sífilis, rubéola, herpes, HIV, citomagalovírus), restrição ao crescimento uterino e mães usuárias de drogas e idade materna. (RESEGUE; PUCCINI; SILVA, 2007).

A identificação desses fatores de risco, juntamente com o conhecimento dos marcos do desenvolvimento, que servem de parâmetro para a identificação precoce de atrasos no desenvolvimento, torna possível realizar um planejamento de intervenções de estimulação ainda nos primeiros anos de vida, reduzindo os efeitos negativos dessa história de riscos. Considerando o fator cultural em que as mães são as principais cuidadoras, seu papel é altamente relevante na representação do ambiente afetivo e de estimulação no desenvolvimento neuropsicomotor. O vínculo afetivo é fortalecido e mantido conforme os bebês respondem às estimulações (com sorrisos, imitações, fala, entre outros comportamentos), como explicam Graciano, Tavano e Bachega (2007), ao enfatizarem a importância de os cuidadores reconhecerem a "linguagem de amor do bebê" para a ligação afetiva durante o desenvolvimento infantil.

Porém, crianças com atraso no desenvolvimento ou com condições atípicas, podem ter respostas mais sutis à estimulação, afetando a percepção desses feedbacks comportamentais por parte da mãe e, consequentemente, enfraquecendo o vínculo (BRASIL, 2016). A forma como a família recebe o diagnóstico da fissura labiopalatina, e posteriormente, como todos os envolvidos vão lidar com essa condição, pode refletir diretamente no desenvolvimento biopsicossocial da criança, já que esta necessita receber uma gama de estímulos externos que são essenciais para que seu desenvolvimento global aconteça de forma positiva (VANZ; RIBEIRO, 2011).

As reações iniciais dos pais ao descobrirem as malformações, são majoritariamente negativas, sejam elas de choque, negação, tristeza, ansiedade, desamparo, culpabilização e estresse psicossocial, às quais, se não forem bem conduzidas, podem favorecer o desenvolvimento de distúrbios emocionais (GRACIANO; TAVANO; BACHEGA, 2007). Segundo Clavarino et al. (2010), os quadros de ansiedade são muito frequentes em mulheres expostas à situações geradoras de estresse elevado, como o nascimento de um filho prematuro, doenças perinatais ou ainda, malformações. 
O estudo desenvolvido por Silva, Rodrigues e Lauris (2017) identificou altos níveis de ansiedade materna nas fases pré e pós-natal, associados à prejuízos no desenvolvimento da criança, como problemas emocionais, cognitivos e comportamentais. Portanto, a saúde mental das mães influencia a relação afetiva, e os problemas emocionais, como ansiedade e depressão materna, se tornam fatores de risco na interação diática, o que pode influenciar significativamente a maneira como ela interage e cuida da sua criança (SILVA; RODRIGUES; LAURIS, 2017). Um fator de proteção contra problemas na saúde mental de mães de crianças com malformações é o apoio da família e/ou do parceiro e acompanhamento profissional humanizado, integrado com a família desde a descoberta da FLP para reabilitação integral da criança (RIBEIRO; SILVEIRA, 2015; MORAES; BUFFA; MOTI, 2009).

A partir do exposto, este estudo objetivou relacionar o desenvolvimento neuropsicomotor, a interação afetivo-social de bebês com FLPNS aos níveis de ansiedade e depressão maternos.

\section{METODOLOGIA}

Esse foi um estudo observacional transversal realizado em um hospital referência na reabilitação de anomalias craniofaciais, no interior do estado de São Paulo. A pesquisa foi aprovada pelo Comitê de Ética da instituição

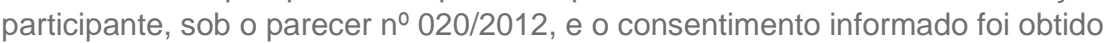
com os pais para a sua participação e do filho. A coleta foi realizada entre março e novembro de 2012.

\section{PARTICIPANTES}

Foram convidadas a participar do estudo 40 díades, mãe-bebê, no berçário do pós-operatório das cirurgias primárias de lábio e/ou palato da instituição participante.

As crianças, de ambos os sexos, com idade de 01 a 12 meses, foram 27 do sexo masculino, provenientes de diferentes regiões do país, e constituindo três grupos de acordo com o tipo de fissura, sendo 16 Gpré, 19 Gtrans e 05 Gpós. A faixa etária das mães foi de 16 anos a 40 anos (Média 26,6 anos), com nível de escolaridade analfabeto à superior completo.

\section{INSTRUMENTOS}

Os instrumentos foram selecionados visando identificar as variáveis demográficas dos participantes, os níveis de ansiedade e depressão maternos, o padrão de desenvolvimento neuropsicomotor e a qualidade da interação afetivo-social das crianças.

\section{VARIÁVEIS DEMOGRÁFICAS}

Anamnese com as mães: para identificação de dados demográficos da mãe e da criança, tais como, idade, ocupação, estado civil, local de origem, antecedentes gestacionais, condições do nascimento, histórico de saúde e tipo de fissura da criança. 
A Escala de Depressão de Beck (BDI) é uma escala auto-aplicável utilizada para avaliação dos estados depressivos (BECK et al., 1982). São 21 itens que categorizam atitudes sintomáticas que representam manifestações de estados depressivo, incluindo atitude, sintomas depressivos e ideação suicida. Neste estudo, foi utilizada a escala de depressão de Beck validada em português no Brasil por Gorenstein e Andrade (1996). A consistência interna (Cronbach's alpha) desta versão para amostra não-clínica foi de 0,81, e 0,88 para a amostra clínica (GORENSTEIN; ANDRADE, 1996).

A Escala de Ansiedade de Beck (BAI) também é uma escala auto-aplicável que indica os níveis de sintomas ansiosos (CUNHA, 2001). São 21 itens que contém sintomas comuns de ansiedade, como sudorese e sentimentos de angústia. Foi utilizada a tradução e validação realizada por Cunha (2001), apresentando consistência interna $\alpha=0,92$.

\section{DESENVOLVIMENTO NEUROPSICOMOTOR INFANTIL}

O Protocolo de Avaliação do Desenvolvimento Global de Crianças no Primeiro ano de Vida (NARDI et al. 2015) é constituído por 51 tarefas, analisadas nas diferentes áreas do desenvolvimento: motor, cognitivo-linguístico e afetivosocial. São avaliadas três faixas etárias: 0-3 meses, 4-8 meses e 9-12 meses. Cada uma delas é composta por 17 marcos do desenvolvimento esperados para a idade do bebê e o resultado final é a soma do número de marcos atingidos. A análise do desempenho é realizada com base na porcentagem de marcos atingidos em relação ao esperado.

\section{QUALIDADE DA INTERAC̣ÃO AFETIVO-SOCIAL}

A Escala de Avaliação da Reação de Retração Prolongada da Criança Pequena (GUEDENEY, 1999) é uma escala clínica utilizada como instrumento de rastreio de patologias psiquiátricas, em crianças no período sensório motor que avalia oito categorias: 1) expressão facial; 2) contato visual; 3) nível geral de atividate; 4) gestos de autoestimulação; 5) vocalizações; 6) vivacidade das reações aos estímulos; 7) relação; e 8) atratividade. A pontuação varia de 1 a 4 em cada um destes domínios, indicando se a qualidade da interação é(0)normal, (1) dúvida sobre o caráter patológico; (2) sinal patológico discreto; (3) evidente para todos os observadores; e (4) intenso. Neste estudo foi utilizada a validação de Assumpção Junior et al. (2002), cuja consistência interna foi de $\alpha=0,8$.

\section{PROCEDIMENTO}

\section{COLETA DE DADOS}

Os dados foram coletados por meio de atendimentos individuais realizados por equipe multidisciplinar composta por uma fisioterapeuta, uma terapeuta ocupacional e uma psicóloga.

Os dados pré, peri e pós natais, que envolveram antecedentes gestacionais, condições do nascimento e histórico de saúde do bebê foi realizada pela psicóloga em situação reservada à anamnese. As atividades para avaliação do desenvolvimento neuropsicomotor foram aplicadas pela fisioterapeuta e pela terapeuta ocupacional, enquanto a psicóloga observava a reação das crianças para o registro e análise dos protocolos da pesquisa. 
A aplicação dos inventários para avaliação dos estados emocionais maternos foi realizada pela psicóloga, em um único atendimento, de aproximadamente 30 minutos com as mães das crianças.

\section{ANÁLISE DOS DADOS}

Os instrumentos normatizados foram corrigidos de acordo com as normas. 0 "Protocolo de Avaliação do Desenvolvimento Global de Crianças no Primeiro ano de Vida" teve seus escores descritos em porcentagens e a "Escala de Avaliação da Reação de Retração Prolongada da Criança Pequena" (ASSUMPÇÃO JUNIOR et al., 2002), que avalia a qualidade da interação, foi atribuída a seguinte classificação pontual: "0 - Sem anormalidades"; "1 - Dúvida sobre o caráter patológico"; "2 - Sinal patológico mais discreto"; "3 - Evidente para todos observadores" e "4 - Intenso".

Os escores totais do "Inventário de depressão de Beck" e "Inventário de ansiedade de Beck", foram obtidos pela somatória dos escores de cada item, que variaram entre 0 e 63 pontos. A classificação seguiu as normas recomendadas pela versão em português validada no Brasil por Cunha (2001), propondo: "1 a 10 pontos = Mínimo"; 11a 19 pontos = Leve"; "20 a $30=$ Moderado"; "31 a 63 = Grave".

Os dados foram tabelados no programa Microsoft Excel e a análise foi realizada no programa Statistica 10. O teste de Kruskal-Wallis foi utilizado na comparação dos grupos. O teste de Qui-quadrado foi utilizado para verificar diferença nos domínios da Escala de Avaliação da reação de retração prolongada da criança pequena. Para a correlação entre as variáveis foi utilizado o teste de Spearman. As análises foram realizadas com nível de significância a 5\%.

\section{RESULTADOS E DISCUSSÃO}

Foram avaliadas 40 crianças de ambos os sexos, classificadas em relação ao sexo e tipo de fissura (Tabela 1). A faixa etária compreendeu entre 0 e 12 meses (M: 6,03 meses; DP: 3,16). A faixa etária materna foi de 16 a 40 anos (M: 26,58 anos; DP: 6,3 , sendo $57,5 \%$ na faixa etária entre 20 e 35 anos. A escolaridade variou do nível analfabeto ao superior completo, sendo que $42,5 \%$ da amostra tinha ensino médio completo.

Tabela 1: Caracterização da amostra em relação ao sexo e ao tipo de fissura

\begin{tabular}{lcccccccc}
\hline & \multicolumn{2}{c}{ Trans-forame } & \multicolumn{2}{c}{ Pré-forame } & \multicolumn{2}{c}{ Pós-forame } & \multicolumn{2}{c}{ Total } \\
\hline & $\mathrm{n}$. & $(\%)$ & $\mathrm{n}$. & $(\%)$ & $\mathrm{n}$. & $(\%)$ & $\mathrm{n}$. & $(\%)$ \\
\hline Masculino & 10 & $25 \%$ & 13 & $33 \%$ & 4 & $10 \%$ & 27 & $68 \%$ \\
\hline Feminino & 9 & $23 \%$ & 3 & $8 \%$ & 1 & $3 \%$ & 13 & $33 \%$ \\
\hline Total & 19 & $48 \%$ & 16 & $40 \%$ & 5 & $13 \%$ & 40 & $100 \%$ \\
\hline
\end{tabular}

No que diz respeito aos fatores pré-natais, o estudo apontou que $37,5 \%$ das mães avaliadas apresentaram intercorrências nesse período. Os relatos envolveram doenças maternas, tabagismo, o uso de medicamentos, álcool e outras drogas. Os medicamentos mais utilizados pelas mães foram os antibióticos (20\%). Outros medicamentos utilizados foram anti-gripal, antialérgico, analgésico, anti-ácido, fármaco anti-hipertensivo e inibidor de contrações uterinas. Neste mesmo período, verificou-se a ocorrência de 
infecção urinária em 22,5\%, pressão alta em 7,5\% e casos de alergias, problemas estomacais e tumores uterinos, com necessidades de repouso no período gestacional.

As intercorrências peri-natais ocorreram em 25\% da amostra e envolveram prematuridade $(7,5 \%)$, baixo peso ao nascer $(5 \%)$ e icterícia $(15 \%)$. Em decorrência disso, as crianças passaram por internações no período pós-natal, sendo $12,5 \%$ na UTI, $10 \%$ na incubadora e $7,5 \%$ no berçário. A necessidade de banho de luz esteve presente em $10 \%$ dos casos. Em relação à amamentação, $25 \%$ dos recém-nascidos tiveram amamentação insuficiente e 15\% utilizou sonda nasogástrica. As doenças infantis evidenciadas foram: resfriados (7,5\%), coqueluche $(2,5 \%)$ e desnutrição $(2,5 \%)$. Uma criança apresentou malformação associada à FLP (sindactilia) e outra, deficiência auditiva.

A análise do Protocolo de Avaliação do Desenvolvimento Global de Crianças no Primeiro ano de Vida indicou que as crianças atingiram em média 84,13\% (DP $=10,43$ ) dos marcos esperados para o desenvolvimento. Quando analisado o desempenho por tipo de fissura, o Gpré teve o desempenho mais baixo, atingindo $82 \%(\mathrm{DP}=9,74)$ dos marcos do desenvolvimento infantil, seguido do Gtrans com 85,71\% (DP = 9,24) e do Gpós, com 91,37\% (DP = 11,73). Apesar destas diferenças, não houve diferença estatisticamente significante entre os grupos ( $p=0,2193)$. O gráfico 1 indica o desempenho das crianças.

\section{Gráfico 1: Desempenho dos bebês no Protocolo de Avaliação do Desenvolvimento Global de Crianças no Primeiro ano de Vida.}

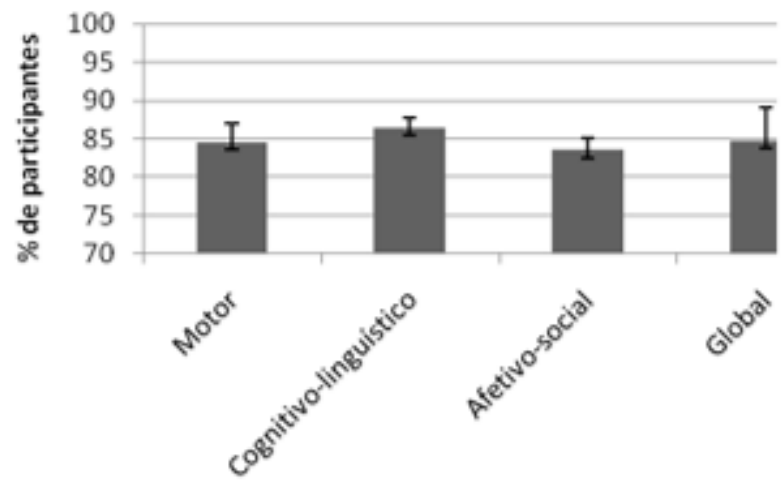

Dominio avaliado

Na análise da Escala de Avaliação da Reação de Retração Prolongada da Criança Pequena a pontuação média foi de 1,92 ( $\mathrm{DP}=2,48)$. Quando analisado por tipo de fissura, o Gtrans teve pior desempenho, com 2,79 pontos ( $D P=3,21$ ), seguido do grupo Gpré com 1,4 (DP = 1,95) e do grupo Gpós, com 1,06 pontos $(\mathrm{DP}=0,93)$. Também não houve diferença estatisticamente significante entre os grupos $(p=0,1968)$. O domínio que mais apresentou dúvidas sobre o caráter patológico foi o de vocalização, seguido de expressão facial $\left(X^{2}=78,11715 ; p=\right.$ ,00). A tabela 2 demonstra os domínios da escala. 
Tabela 2 - Representação dos domínios da reação de retração prolongada.

\begin{tabular}{|c|c|c|c|c|c|}
\hline Comportamento & $\begin{array}{c}\text { Normal } \\
(\%)\end{array}$ & $\begin{array}{c}\text { Dúvida } \\
\text { sobre o } \\
\text { caráter } \\
\text { patológico } \\
(\%) \\
\end{array}$ & $\begin{array}{c}\text { Sinal } \\
\text { patológico } \\
\text { discreto } \\
(\%)\end{array}$ & $\begin{array}{c}\text { Evidente } \\
\text { para todos } \\
\text { observadores } \\
(\%)\end{array}$ & $\begin{array}{c}\text { Intenso } \\
(\%)\end{array}$ \\
\hline Expressão facial & 72,5 & 22,5 & 2,5 & 2,5 & 2,5 \\
\hline Contato visual & 90,0 & 10,0 & 0,0 & 0,0 & 0,0 \\
\hline Atividade corporal & 87,5 & 12,5 & 0,0 & 0,0 & 0,0 \\
\hline $\begin{array}{l}\text { Gestos de auto } \\
\text { estimulação }\end{array}$ & 92,5 & 7,5 & 0,0 & 0,0 & 0,0 \\
\hline Vocalizações & 42,5 & 42,5 & 2,5 & 7,5 & 7,5 \\
\hline Vivacidade & 85,0 & 15,0 & 0,0 & 0,0 & 0,0 \\
\hline Relação & 87,5 & 10,0 & 2,5 & 0,0 & 0,0 \\
\hline Atratividade & 90,0 & 10,0 & 0,0 & 0,0 & 0,0 \\
\hline
\end{tabular}

Em relação à depressão e ansiedade materna, foram prevalentes os valores mínimos de depressão (72,5\%) e ansiedade (60\%). A depressão foi identificada em $7,5 \%$ das mães (moderado e grave), enquanto a ansiedade foi detectada em $12,5 \%$ (moderado e grave), como indica o Gráfico 2. Em relação ao tipo de fissura também não houve diferença estatisticamente significante entre os grupos (BDI, $p=0,6570 ; B A I, p=0,9957$ ).

\section{Gráfico 2 - Desempenho das mães na BAl e na BDI.}

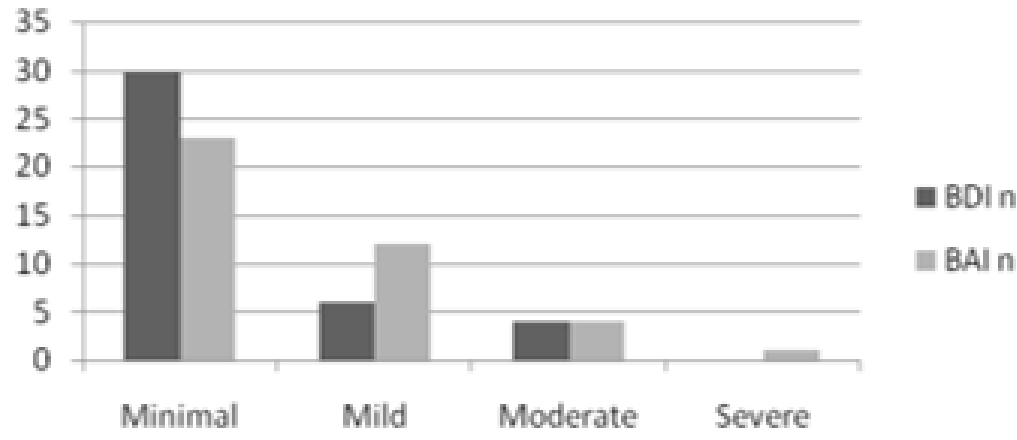

O desempenho das mães nas escalas de humor foi correlacionado ao desempenho das crianças nas escalas de desenvolvimento, sendo identificada correlação entre ansiedade materna e nível geral de atividade $(r=-0,31)$, gestos de autoestimulação $(r=0,40)$ e a relação da criança com o observador $(r=-0,31)$, conforme apresentado na Tabela 3. 
Tabela 3: Correlação entre os domínios avaliados

\begin{tabular}{lrr}
\hline & $\begin{array}{c}\text { Depressão } \\
\text { materna }\end{array}$ & $\begin{array}{c}\text { Ansiedade } \\
\text { materna }\end{array}$ \\
\hline Expressão Facial & 0,12 & 0,10 \\
\hline Contato ocular & $-0,02$ & $-0,13$ \\
\hline Nível geral de atividade & $-0,22$ & $-\mathbf{0 , 3 1 ^ { * }}$ \\
\hline Gestos de auto estimulação & 0,23 & $\mathbf{0 , 4 0 ^ { * }}$ \\
\hline Vocalizações & $-0,05$ & 0,10 \\
\hline Vivacidade das reações aos & $-0,10$ & $-0,05$ \\
\hline estímulos & $-0,22$ & $-\mathbf{0 , 3 1 ^ { * }}$ \\
\hline Relação & 0,15 & 0,07 \\
\hline Atratividade & $-0,05$ & $-0,13$ \\
\hline Desenvolvimento psicomotor & & \\
\hline *valores de $\mathrm{p} \leq 0,05$. & &
\end{tabular}

As crianças com fissura labiopalatina na idade de 0 a 1 ano foram avaliadas quanto ao desenvolvimento dos aspectos motor, cognitivo-linguístico e afetivosocial, e os resultados comparados a aspectos afetivos das respectivas mães. São escassos os estudos que demonstram perfis do desenvolvimento cognitivo, afetivo e psicomotor de crianças com FLPNS. A utilização de instrumentos de medida como as escalas de desenvolvimento são importantes na mensuração das aquisições de habilidades que o bebê adquire conforme o crescimento, pois é importante que se detecte $o$ atraso no desenvolvimento motor o mais precocemente possível, para que seja possível intervir precocemente de modo a reduzir riscos e efeitos negativos (PANCERI; PEREIRA; VALENTINI, 2017). Observou-se que a maioria da amostra é acometida pela fissura transforame e é do sexo masculino. A fissura transforame é aquela de maior incidência no Brasil e no exterior (GARDENAL et al., 2011; MARTELLI JUNIOR et al., 2007). Este tipo de fissura é também o mais grave, em virtude da extensão anatômica da malformação. Os pacientes que apresentam esta malformação necessitam de tratamento prolongado e com maior quantidade de correções cirúrgicas para uma adequada reabilitação estética anatomo funcional e psicossocial (SILVA FILHO, 1998). A prevalência do sexo masculino corrobora achados da literatura pesquisada, que indicam que os meninos são os mais acometidos pelas fissuras pré e transforame, ao passo que as meninas, são mais acometidas pelas fissuras pós-forame (FREITAS et al., 2012).

A faixa etária predominante na amostra, entre 4 e 8 meses, justifica-se pelo período em que são realizadas as cirurgias de reparação primárias de lábio e palato, respectivamente, queiloplastia e palatoplastia, no hospital de referência do estudo (RAZERA et al., 2016). As primeiras cirurgias são realizadas a partir do terceiro mês e este é o período desejável para o início do tratamento das fissuras.

As intercorrências relatadas no período pré-natal envolveram doenças, uso de medicamentos, álcool e drogas, como a nicotina. A literatura indica que tanto fatores genéticos, quanto ambientais estão envolvidos na etiologia das fissuras 
e dentre os reportados pelas mães, o tabagismo se apresentou como fator de risco para a fissura (COUTINHO, 2009).

De acordo com o Ministério da Saúde (BRASIL, 2001), a faixa etária materna entre 16 e 40 anos no período de gestação, é considerada de baixo risco. No presente estudo, a média de idade foi de 27 anos, não sendo a idade, portanto, fator de risco para a gravidez, já que idades extremas, como abaixo de 16 e acima de 35 anos (LAOPAIBOON et al., 2014), são consideradas faixas de risco para o desenvolvimento do bebê.

$\mathrm{Na}$ avaliação dos bebês, foi observado que a amostra apresentou recursos satisfatórios no desenvolvimento psicomotor, observada na qualidade do olhar, no balbucio e nos movimentos intencionais, em respostas objetivas aos estímulos do ambiente. Quando comparado o desempenho em relação ao tipo de fissura, não foram encontradas diferenças significantes entre os grupos. $\mathrm{Na}$ escala de ação de retração não houve indicativo de prejuízos na qualidade da interação com o observador. Estes achados remetem às condições favoráveis da criança interagir com o meio e ter ações responsivas aos estímulos sensoriais e afetivos positivos durante o primeiro ano de vida, que, segundo Tronick e Beeghly (2011), é um importante preditor de seu ajustamento psicológico nas idades posteriores em diferentes contextos. Dentre as justificativas para este achado, a literatura traz que um ambiente familiar acolhedor promove na criança o desenvolvimento de repertórios sociais eficazes desde as intenções rudimentares do bebê, período no qual ocorre a construção de significado do mundo exterior (TRONICK; BEEGHLY, 2011; PAPALIA et al., 2001).

Os estudos sobre depressão e ansiedade nas mães de crianças com fissura labiopalatina são escassos e sua prevalência não é determinada., porém, estudos recentes apontam para a possibilidade de correlacionar problemas de comportamento em crianças com fissura labiopalatina de idade pré-escolar e ansiedade materna, considerado como fator de risco para a evolução de comportamentos inadaptativos (SILVA; RODRIGUES; LAURIS, 2017). Neste estudo, as escalas aplicadas nas mães foram bem aceitas e facilmente compreendidas, sendo que as mães avaliadas não apresentaram sintomatologia depressiva e de ansiedade com uma condição de distúrbio instalada, ou seja, não apresentaram, na sua maioria, uma patologia de base depressiva ou ansiosa generalizada. Esses achados corroboraram os encontrados por Montirosso et al. (2012), no qual foi avaliado o perfil emocional de mães de bebês com FLPNS, em que o BDI foi autoaplicado à 25 mães de crianças com FLPS e 25 mães compondo grupo controle. Mesmo não tendo sido encontradas diferenças estatisticamente significantes, identificou que as mães de crianças com FLPNS manifestavam sintomas depressivos, mas ocultados no autorrelato, o que sugeriu que essas mães ignoravam ou tenderam a minimizar estados emocionais negativos. Por outro lado, os baixos níveis de depressão e ansiedade em mães, evidenciados no estudo, poderiam ser o resultado do tratamento humanizado oferecido a elas e seus filhos, além do suporte psicológico e da convivência em grupos operacionais na rotina institucional, que, de acordo com Ribeiro e Silveira (2015), são mecanismos de suporte essenciais para o bemestar do paciente e de seus familiares, minimizando os efeitos negativos da hospitalização na saúde mental materna (MORAES; BUFFA; MOTI, 2009).

Ao relacionar os níveis de depressão e ansiedade maternos com o desenvolvimento do bebê, foi encontrada correlação positiva entre gestos de autoestimulação e os níveis de ansiedade, indicando que filhos de mães ansiosas apresentam maiores sinais de retração nesse domínio. Mães ansiosas tendem a dificultar a interação com o bebê, uma vez que há um descompasso 
entre as próprias demandas decorrentes de estados ansiógenos, e as respostas do bebê (reações adaptativas). Desta forma, cria-se um espaço favorável para comportamentos de autoestimulação da criança, visando a gratificação de suas necessidades afetivas e sociais.

Uma das limitações do presente estudo, cujo objetivo foi relacionar aspectos motores, cognitivo-linguístico e afetivo-social aos afetivos das mães, foi a amostra restrita de mães com índices significativos de ansiedade e depressão, bem como, a participação maior de crianças com bom desenvolvimento. Isso pode ser um fator limitante à análise estatística. Desta maneira, sugere-se que sejam feitos estudos com mães diagnosticadas com ansiedade e depressão para melhor identificação de relação entre os aspectos do desenvolvimento infantil e a saúde mental materna.

\section{CONSIDERAÇÕES FINAIS}

Um contingente representativo de bebês com FLPNS apresentou desenvolvimento psicomotor e interações adequadas correspondente à faixa etária. As mães apresentaram baixos níveis de depressão e ansiedade, contrariando a literatura consultada. Não houve correlações significantes entre o desenvolvimento psicomotor e o estado de saúde materno. Na qualidade das interações, foi identificada correlação moderada entre ansiedade materna e gestos de autoestimulação do bebê. Desta forma o estudo concluiu que o estado de humor materno não interferiu no desenvolvimento psicomotor do bebê, porém, os filhos de mães mais ansiosas tenderam a maiores índices de gestos de autoestimulação.

\section{REFERÊNCIAS}

ASSUMPCAO JR, F. B. et al. Escala de avaliação da reação de retração no bebê: Um estudo de validade. Arq. Neuro-Psiquiatr., São Paulo, v. 60, n. 1, p. 56-60, Mar, 2002.

BECK, T. A. et al. Terapia cognitiva da depressão. Rio de Janeiro: Zahar, 1982.

BRASIL. Ministério da Saúde. Secretaria de Políticas de Saúde. Área Técnica de Saúde da Mulher: Parto, aborto e puerpério: assistência humanizada à mulher. Brasília: Ministério da Saúde, 2001.

BRASIL. Ministério da Saúde. Secretaria de Atenção à Saúde. Diretrizes de estimulação precoce: crianças de zero a 3 anos com atraso no desenvolvimento neuropsicomotor decorrente de microcefalia. Ed. Brasília: Ministério da Saúde, 2016.

BRAZELTON, T. Berry. O Grande Livro da Criança: o desenvolvimento emocional e do comportamento durante os primeiros anos. 9. ed. Lisboa: Editorial Presença, 2006

CLAVARINO, A. M. et al. Maternal anxiety and attention problems in children at 5 and 14 years. Journal of Attention Disorders, n.13, p. 658-667, 2010. 
COUTINHO, A. L. F. et al. Perfil epidemiológico dos portadores de fissuras orofaciais atendidos em um Centro de Referência do Nordeste do Brasil. Rev. Bras. Saude Mater. Infant., Recife, v. 9, n. 2, p. 149-156, Jun, 2009.

CUNHA, J. A. Manual da versão em português das Escalas Beck. São Paulo: Casa do Psicólogo, 2001.

FREITAS, J.A.S. et al. Rehabilitative treatment of cleft lip and palate: experience of the Hospital for Rehabilitation of Craniofacial Anomalies-USP (HRAC-USP) - part 2: pediatric dentistry and orthodontics. Journal of Applied Oral Science (Impresso), v. 20, p. 268-281, 2012.

GARDENAL, M. et al. Prevalências das fissuras orofaciais diagnosticadas em um serviço de referência em casos residentes no estado do Mato Grosso do Sul. Arq. Inter. Otorrinolaringol. São Paulo, v.15, n.2, p.133-141, abr/mai/junho, 2011.

GARIB, D.G. et al. Etiologia das más oclusões: perspectiva clínica (parte III) - Fissuras labiopalatinas. Rev Clin Ortod Dental Press. v. 09, n. 4, p. 30-6, 2011.

GORENSTEIN C., ANDRADE L. Validation of a Portuguese version of the Beck Depression Inventory and the State-Trait Anxiety Inventory in Brazilian subjects. Braz J Med Biol Res. v. 29: p. 453-457, 1996.

GRACIANO, M. I.; TAVANO, L.D.; BACHEGA, M.I. Aspectos psicossociais da Reabilitação. In: TRINDADE, I.E.K.; SILVA FILHO, O..G. Fissuras Labiopalatinas: uma abordagem interdisciplinar. Rio de Janeiro: Santos, 2007. p. 311-33.

GROSEN, D. et al. Risk of oral clefts in twins. Epidemiology, v. 22 n.3, p.313-319, 2011.

GUEDENEY, A. De la reaction precoce et durable de retrait a la depressionchez le jeune enfant. Neuropsychiatr Enfance Adolesc. v. 47, p. 63$71,1999$.

LAOPAIBOON, M. et al. Advanced maternal age and pregnancy outcomes: a multicountry assessment. BJOG. v.21, p.49-56, 2014.

MARTELLI JUNIOR, H. et al. Prevalence of nonsyndromic oral clefts in a reference hospital in Minas Gerais State, between 2000-2005. Braz Oral Res. v.21 n.4 p. 314-17, 2007.

MATTA, I. Psicologia do desenvolvimento e aprendizagem. Lisboa: Universidade Aberta, 2001.

MONTIROSSO, R. et al. Level of NICU quality of developmental care and neurobehavioral performance in very preterm infants. Pediatrics, v. 129 n.5, p. 1129-1137, 2012. 
MORAES, M. C. A. F.; BUFFA, M. J. M. B.; MOTTI, T. F. G. As atividades expressivas e recreativas em crianças com fissura labiopalatina hospitalizadas: visão dos familiares. Rev. bras. educ. espec., Marília, v. 15, n. 3, p. 453-470. http://dx.doi.org/10.1590/S1413-65382009000300009.

NARDI, C. G. A. et al. Bebês com Sequência de Pierre Robin: saúde mental materna e interação mãe-bebê. Estudos de psicologia (Campinas), Campinas. v. 32, n. 1, p. 129-140, Mar, 2015.

ORGANIZAÇÃO PAN-AMERICANA DA SAÚDE. Manual para vigilância do desenvolvimento infantil no contexto da AIDPI. Washington, D.C., 2005.

PANCERI, C.; PEREIRA, K.R.G.; VALENTINI, N. C. 2017 A intervenção motora como fator de prevenção de atrasos no desenvolvimento motor e cognitivo de bebês durante o período de internação hospitalar. Cadernos Brasileiros de Terapia Ocupacional. v.25 n.3 p. 469-479, 2017. https://doi.org/10.4322/2526-8910.ctoAO0977

PAPALIA, D.; OLDS, S. W.; FELDMAN, R. D. O mundo da criança. 8. ${ }^{\text {a }}$ edição. Lisboa: McGraw-Hill, 2001.

RAZERA, A. P. R. et al. Vídeo educativo: estratégia de treinamento para cuidadores de crianças com fissura labiopalatina. Acta Paul Enferm. v.29, n.4, p.430-438, 2016.

RESEGUE, R.; PUCCINI, R. F.; SILVA, E. M. K. S. Fatores de risco associados a alterações no desenvolvimento da criança. Pediatria, [S.I.], v. 29, n. 2, p. 117-128, 2007.

RIBEIRO, D. G.; PEROSA, G. B.; PADOVANI, F. H. P. Fatores de risco para o desenvolvimento de crianças atendidas em Unidades de Saúde da Família, ao final do primeiro ano de vida: aspectos sociodemográficos e de saúde mental materna. Ciência e Saúde Coletiva. v.19 n.2, p.2-26, 2014.

RIBEIRO, I.; SILVEIRA, M.G.C.C. Humanização Hospitalar no Sistema Único de Saúde. Revista Interdisciplinar Ciências e Saúde, Ago-out, 2015.

SILVA FILHO, O. G. et al. Abordagem ortodôntica ao paciente com fissura unilateral completa de lábio e palato. Ortodontia, São Paulo, v. 31, n. 3, p. 32-44, set./dez. 1998

SILVA, F.; RODRIGUES, O. M. P. R.; LAURIS, J. R. P. Ansiedade Materna e Problemas Comportamentais de Crianças com Fissura Labiopalatina. Psicol. cienc. prof., Brasília, v. 37, n. 2, p. 318-334, June, 2017. http://dx.doi.org/10.1590/1982-3703000682016.

TRINDADE, I. E.K.; SILVA FILHO, O.G. Fissuras Labiopalatinas: uma abordagem interdisciplinar. Rio de Janeiro: Ed. Santos, 2007.

TRONICK, E.; BEEGHLY M. Infants' Meaning-Making and the Development of Mental Health Problems. Am Pshycol. v.66 n.2, p.107-119, Feb-Mar. 2011. 
UNICEF. Early Childhood Development: the key to a full and productive life. 2015. Disponível em: <http://www.unicef.org/dprk/ecd.pdf>. Acesso em: 3 maio 2018.

VANZ, A. P.; RIBEIRO, N. R. R. Escutando as mães de portadores de fissuras orais. Rev. esc. enferm. USP, São Paulo, v. 45, n. 3, p. 596-602, June,2011.

Recebido em: 01-06-2018

Aceito em: 03-10-2019 